\title{
Identifying genetic determinants of complex phenotypes from whole genome sequence data
}

\author{
George S. Long ${ }^{1}$, Mohammed Hussen ${ }^{1}$, Jonathan Dench ${ }^{1}$ and Stéphane Aris-Brosou ${ }^{1} 2^{*}$ (i)
}

\begin{abstract}
Background: A critical goal in biology is to relate the phenotype to the genotype, that is, to find the genetic determinants of various traits. However, while simple monofactorial determinants are relatively easy to identify, the underpinnings of complex phenotypes are harder to predict. While traditional approaches rely on genome-wide association studies based on Single Nucleotide Polymorphism data, the ability of machine learning algorithms to find these determinants in whole proteome data is still not well known.

Results: To better understand the applicability of machine learning in this case, we implemented two such algorithms, adaptive boosting (AB) and repeated random forest (RRF), and developed a chunking layer that facilitates the analysis of whole proteome data. We first assessed the performance of these algorithms and tuned them on an influenza data set, for which the determinants of three complex phenotypes (infectivity, transmissibility, and pathogenicity) are known based on experimental evidence. This allowed us to show that chunking improves runtimes by an order of magnitude. Based on simulations, we showed that chunking also increases sensitivity of the predictions, reaching 100\% with as few as 20 sequences in a small proteome as in the influenza case (5k sites), but may require at least 30 sequences to reach $90 \%$ on larger alignments (500k sites). While RRF has less specificity than random forest, it was never $<50 \%$, and RRF sensitivity was significantly higher at smaller chunk sizes. We then used these algorithms to predict the determinants of three types of drug resistance (to Ciprofloxacin, Ceftazidime, and Gentamicin) in a bacterium, Pseudomonas aeruginosa. While both algorithms performed well in the case of the influenza data, results were more nuanced in the bacterial case, with RRF making more sensible predictions, with smaller errors rates, than $A B$.

Conclusions: Altogether, we demonstrated that ML algorithms can be used to identify genetic determinants in small proteomes (viruses), even when trained on small numbers of individuals. We further showed that our RRF algorithm may deserve more scrutiny, which should be facilitated by the decreasing costs of both sequencing and phenotyping of large cohorts of individuals.
\end{abstract}

Keywords: Influenza virus, Pseudomonas aeruginosa; Machine learning, Genome-wide association study, Drug resistance

\section{Background}

An overarching goal in biology is to predict an individual's phenotype from its genotype, in a given environment [1], or from a given genetic makeup [2]. One possibility is to find the genetic determinants of each phenotype of interest - which is what genome-wide association studies

*Correspondence: sarisbro@uottawa.ca

'Department of Biology, University of Ottawa, Ottawa, Ontario, Canada ${ }^{2}$ Department of Mathematics and Statistics, University of Ottawa, Ottawa, Ontario, Canada
(GWAS's) have endeavored to achieve over the past ten years [3]. At their foundation, GWAS's rely on the analysis of millions of variants in the genome, without any prior knowledge of their involvement with a particular phenotype, over a sample of unrelated individuals: as such, GWAS's are often qualified of performing an "unbiased scan of the genome" [4]. While GWAS's have some limitations that may be shared by alternative approaches (they assume that common diseases are caused by common variant [4], which may result in failing to explain most of 
the phenotypic variance [5]), their main issue may lie in their reliance on Single Nucleotide Polymorphism (SNP) data, that only offer a partial snapshot of the genome, and that may not even contain the causative agents of the phenotype under study. The immediate alternative to using SNP data would be resort to high throughput sequencing technology, and work directly with entire proteome data [6], which are predicted to replace SNP data [3]. However, changing from focal SNP's to whole proteome information will radically increase the number of tests to be performed, and might lead to statistical complications in controlling false discovery, as acknowledged at the very outset of GWAS [7]. Recently, it was suggested that machine learning could be used to predict susceptibility to some cancers in humans [8]. Building upon these developments, we here hypothesized that machine learning is able to go beyond predicting genomic inheritance within a cohort of individuals, by predicting the genetic determinants of particular phenotypes using proteome data.

A large number of machine learning approaches exist [9], and are gaining popularity in biology [10, 11]. For instance, in the work just cited above, the authors resorted to neural networks [8]. While some statisticians claim that adaptive boosting (AB) [12] is the "best off-the-shelf classifier in the world" $[9,13]$, it is probable that no single classifier can be considered perfect in all situations - a situation known as the no free lunch theorem [14]. Technically, AB relies on an iterated process where linear decisions are fitted in the space of proteomic features (amino acid positions in a protein alignment). At each iteration, individual observations that were misclassified in the previous iteration are emphasized, so that the algorithm learns from past errors. While each iteration typically leads to a weak classifier that is just a bit better than chance, the final classifier takes advantage of combining these weak classifiers to improve (boost) their performance and construct a strong one [12], i.e. a classifier with an accuracy that can come close to 99\% [15]. While our recent success with $\mathrm{AB}$ [16] prompted us to further investigate this algorithm in the context of proteome data, we also aimed at comparing its performance with more popular algorithms, such as random forests [17] (RF). The RF algorithm is essentially based on decision trees [18], combined with a bootstrap procedure (bagging) aimed at increasing the stability of the predictions, and a random subsetting of predictors to decorrelate the bagged trees, that are then averaged to produce the final classifier.

As any supervised learning algorithm, $A B$ and $R F$ are trained on labeled data, i.e. data for which the correct assignments are known. In our case, the proteome data coming from an organism for which drug resistance (the label / phenotype) is known. Before training the algorithm, the labeled data are usually split into two subsets, a train and a test data set, where only the former is used to train the classifier. This trained classifier is then tested on the independent test data set, to validate its performances, and can then be used to classify new individuals, which were neither part of the train nor of the test data sets, as being "drug resistant" or not, based solely on their proteomic information. Here however, our goal is slightly different: we are not interested in classifying individuals, but in finding the most important features (again, these are the mutations at particular sites) that the algorithm is learning from to correctly classify individuals. This is why training will be done on the entire data, rather than splitting them as train and test data sets. More specifically, during the learning process, features (sites) are ranked by decreasing importance in fitting the final model [19]. The algorithm weighs the influence of each feature based on their relative importance during the creation of the model [20], and thus uses only the most important sites. The end result is that we have not merely a model for predicting phenotype, but a means of identifying a ranked list of the most important features [10] that determine a particular phenotype.

To assess the ability of machine learning algorithms to predict the genetic determinants of particular phenotypes, we implemented two such algorithms, AB and a modified RF, and compared their performance in two microbes, one for which most of these determinants are known (the Influenza A virus), and one for which little is known (the Pseudomonas aeruginosa bacterium). In both cases, we retrieved complete proteome sequences of phenotyped individuals. These phenotypes pertained either to their capacity to infect a human host (influenza), or their resistance to particular antibiotics (pseudomonas). Although these two biological systems are very different, both are expected to have a highly complex genetic basis: the molecular determinants of influenza virulence and pathogenesis can span its entire genome [21, 22], and bacterial drug resistance can involve many distinct mechanisms [23]. We took advantage of an influenza database backed by experimental validations [24], and of a recent study of P.aeruginosa genomics [25], to train both algorithms. We modified both original algorithms to make them amenable to analyzing whole proteome data sets, and altered the RF algorithm to further stabilize its predictions by introducing a Repeated Random Forest (RRF) algorithm. We then evaluated the performance of these algorithms with respect to either experimental validations (influenza), or both gene annotations and cross-validations (pseudomonas). We discussed the advantages and limitations of our modified algorithms in identifying the genetic determinants of complex phenotypes from whole genome sequence data. 


\section{Results and discussion}

Determination of the RRF thresholds on the influenza data

The influenza data represent our gold standard here, as we can run our machine learning algorithms on them, and compare the model predictions with experimental evidence (Table 1). Because both algorithms ( $A B$ and RRF) essentially return a list of sites by decreasing importance, we can use the influenza data to determine optimal thresholds for predicting genuine genetic determinants. Two thresholds were employed here: the percentile and the consensus thresholds ( $\mathrm{AB}$ only has the former). This was done by maximizing the number of true positives, i.e. (i) sites that are found in at least a certain number of RF runs of the RRF (our "consensus threshold") - given of course that these sites are backed by experimental evidence in the case of the influenza data (Table 1) - and (ii) sites that have a high importance or mean Gini index (our "percentile threshold"; Figure S1 in Additional file 1). By varying both thresholds simultaneously, we determined

Table 1 Sensitivity of the algorithms on the analysis of the influenza data

\begin{tabular}{|c|c|c|c|c|}
\hline Site & $A B$ & RRF & Phenotype & References \\
\hline PB2 9 & $\checkmark$ & $\checkmark$ & Infectivity & {$[26]$} \\
\hline PB2 105 & $\checkmark$ & $\checkmark$ & Pathogenicity/Infectivity & {$[27]$} \\
\hline PB2 339 & $\checkmark$ & $\checkmark$ & Infectivity & {$[28,29]$} \\
\hline PB2 391 & $\checkmark$ & & Transmissibility & {$[30]$} \\
\hline PB2 627 & $\checkmark$ & $\checkmark$ & Infectivity & {$[31]$} \\
\hline PB2 667 & $\checkmark$ & & Infectivity & {$[32]$} \\
\hline PB1 215 & $\checkmark$ & $\checkmark$ & Pathogenicity & {$[33]$} \\
\hline PB1 375 & & $\checkmark$ & Pathogenicity & {$[34]$} \\
\hline PB1 757 & $\checkmark$ & & Infectivity & {$[35]$} \\
\hline HA 163 & & $\checkmark$ & Pathogenicity/Infectivity & {$[36]$} \\
\hline HA 212 & & $\checkmark$ & Pathogenicity/Infectivity & {$[37]$} \\
\hline HA 246 & $\checkmark$ & $\checkmark$ & Transmissibility & {$[38]$} \\
\hline HA 536 & & $\checkmark$ & Infectivity & {$[39]$} \\
\hline NP 400 & & & Pathogenicity & {$[40]$} \\
\hline NA 49 & & $\checkmark$ & Transmissibility & {$[41]$} \\
\hline NA 75 & & & Transmissibility & {$[42]$} \\
\hline M2 31 & & $\checkmark$ & Pathogenicity/Infectivity & {$[41]$} \\
\hline NS1 127 & & & Pathogenicity & {$[43]$} \\
\hline NS1 195 & & & Transmissibility/Infectivity & {$[44]$} \\
\hline NS1 212 & & $\checkmark$ & Pathogenicity/Infectivity & {$[45]$} \\
\hline
\end{tabular}

This table lists the genes and amino acid positions known to be involved in the three phenotypes studied here, and which one of these were rediscovered by our algorithms. For $A B$, chunk sizes of 75,125 , and 175 were used to calculate the importance values of each site for adaptive boosting. An importance threshold of 1 was used to determine whether a site was a potential genetic determinant. For RRF chunk sizes of 80,125 , and 175 were used with a threshold of the $90^{\text {th }}$ percentile and a $60 \%$ consensus. Data on experimental validations are from the Influenza Research Database [24]. Genes are ordered by segment size. See Figs. 2 and 3 for the specificity of these algorithms that using a consensus threshold around $50 \%$ and the $90^{\text {th }}$ percentile (top 10\% important sites) maximized the number of true positives while minimizing the false positives (Additional file 1: Figures S2-S11). Increasing the percentile threshold to $95 \%$ dramatically increased false negatives, while decreasing it to the $85^{\text {th }}$ percentile only led to more false positives. Likewise, we found that increasing the consensus threshold only decreased the proportion of false positives, without affecting sensitivity. We henceforth used these two RRF thresholds (50\% consensus, $90^{\text {th }}$ percentile).

\section{Chunking improves runtimes, minimally affecting site ranking}

As these algorithms can have large computational requirements for whole proteome analyses ( $\mathrm{AB}$ in particular), we implemented a chunking algorithm (Additional file 1: Figure S12). For this, each alignment was subdivided into smaller alignments (chunks), on which each ML algorithm was run in a first pass to determine a set of positions of interests (AB: those with importance > 1.5; RRF: those with a Gini index $>0.075)$. A second pass ran the same ML algorithm on the entire set of positions of interests to determine the final important sites or selected features over the entire data set. The consensus and percentile thresholds described above are then applied to produce the list of genetic determinants (Additional file 1: Figure S1).

To determine how chunking affected both the runtimes and the predictions of the machine learning algorithms on the influenza data, 20 chunk sizes were compared, ranging from 75 (or 80 in the case of RRF) to 175 by increments of five. Being much faster than $A B$, the RRF algorithm was further tested beyond the initial 20 chunks, up until the sequence alignment was analyzed in full. This was repeated for each of the three influenza phenotypes. As expected from its associated increased memory requirements, increasing chunk sizes also increased runtimes exponentially for both AB (Fig. 1a) and RRF (Fig. 1b). Smaller chunk sizes reduced runtimes by about an order of magnitude ( $1 \log _{10}$ unit) for both $\mathrm{AB}$ and RF. More specifically, an analysis of covariance (ANCOVA) showed that runtimes were similarly affected across all phenotypes in $\mathrm{AB}(P=0.855)$. However, while the slopes of these regressions were similar, the analyses for the pathogenicity phenotype ran the slowest (intercept: 3.85 ; $P=1.12 \times 10^{-5}$ ), followed by transmissibility (intercept: $2.68 ; P=9.26 \times 10^{-7}$ ) and infectivity (intercept: 2.35; $\left.P=4.61 \times 10^{-6}\right)$. Similar results were observed with RRF, where again all slopes were similar $(P=$ 0.973 ), pathogenicity ran the slowest (intercept: $1.175, P<$ $2.00 \times 10^{-16}$ ), followed by infectivity (intercept: 1.174 , $P<2.00 \times 10^{-16}$ ) and transmissibility (intercept: 1.168 , $\left.P<2.00 \times 10^{-16}\right)$. As the feature space was the same (the 

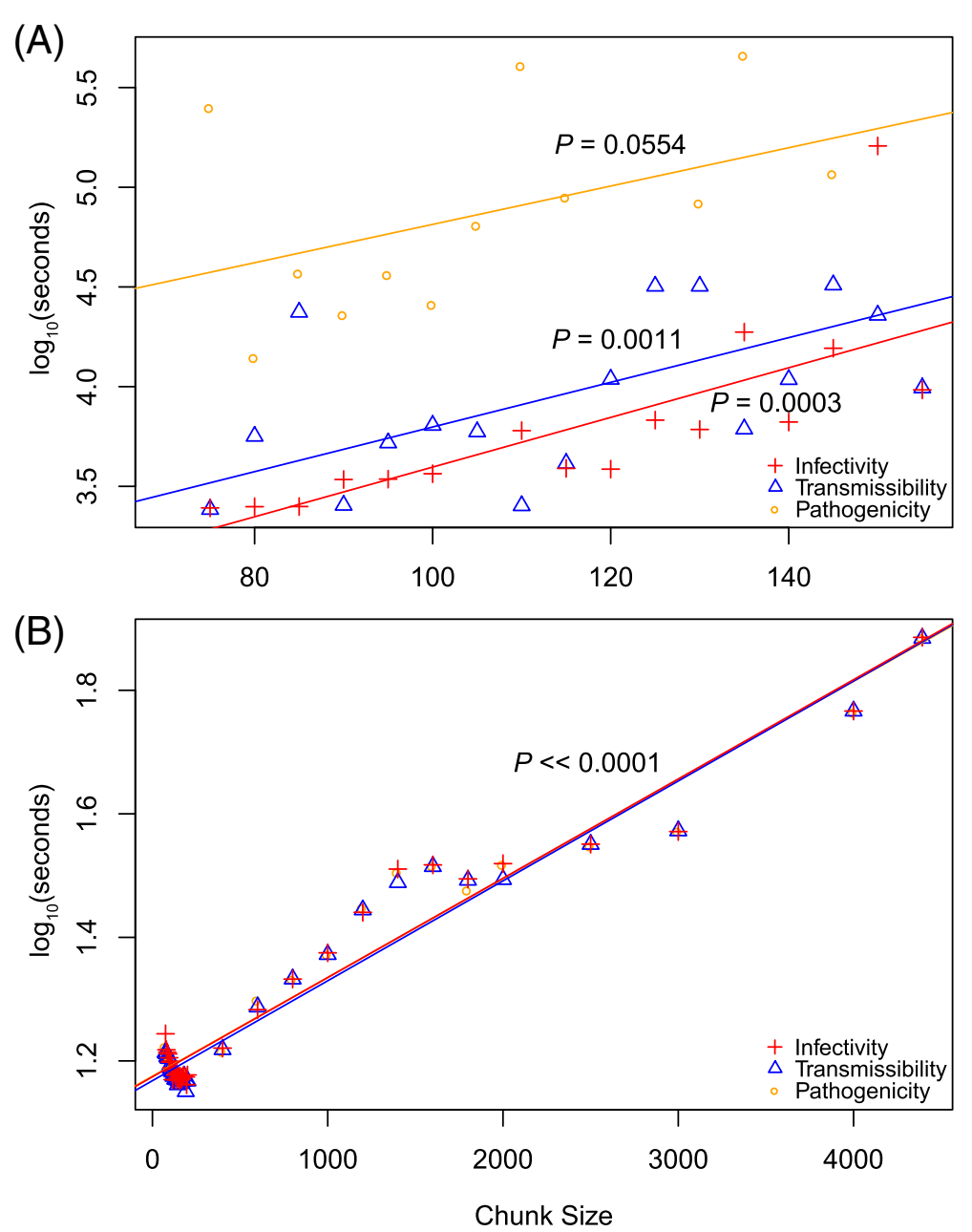

Fig. 1 Impact of chunk size on the runtime of the machine learning algorithms for the influenza data. Runtimes for infectivity (red), transmissibility (blue), and pathogenicity (orange) are shown for $\mathrm{AB}(\mathbf{a})$ and $\operatorname{RRF}(\mathbf{b})$. While each data point is based on a single run, run-to-run variability is taken into account by performing linear regressions (solid lines); their $P$-values are also shown

same amino acid alignment), it must be the distribution of the phenotypes (labels) that impacted runtimes. However, base runtimes (i.e., their intercepts) did not increase due to class imbalance, as the most imbalanced phenotype (transmissibility; Table 5) had an intermediate intercept (Fig. 1a). Finally, note that RRF was actually so fast at small chunk sizes that there was a significant overhead associated with our parallelization of the algorithm (Methods), as linear models fitted to only chunk sizes less than 200 were highly significant $\left(P<2.00 \times 10^{-16}\right)$, with negative slopes (Fig. 1b).

Not only were runtimes significantly and similarly reduced by chunking across phenotypes and algorithms, but differences in terms of which amino acids were predicted to be the most important were also similar (Figs. 2-3; see Venn diagrams in insets, and distributions of importance values for the largest chunk size). In the case of $A B$, the six most important sites determining infectivity at the smallest chunk size (75) were among the top fifteen sites at intermediate (125) and largest (175) chunk sizes (Fig. 2a). Furthermore, the top site, HA 108, was the most important at all of these chunk sizes, while PB2 627 and 667 were always ranked second or third. The RRF results showed a similar pattern in terms of which sites were the most important (Fig. 3). In the case of infectivity, the four top sites (HA 108, HA 536, NA 84 and NA 340) were consistently the most important. After the fourth site, a large drop in importance was observed, suggesting that limited information was available at those sites. But while this drop was also observed for transmissibility at extreme chunk sizes, it was not observed anywhere else, suggesting that the first large drop in ranked importance should not be used to evaluate the relative merit of these predictions. As expected from its repeated nature, the RRF predictions were more stable than those under $\mathrm{AB}$ across chunk sizes (compare Venn insets in Figs. 2 and 3, respectively). 

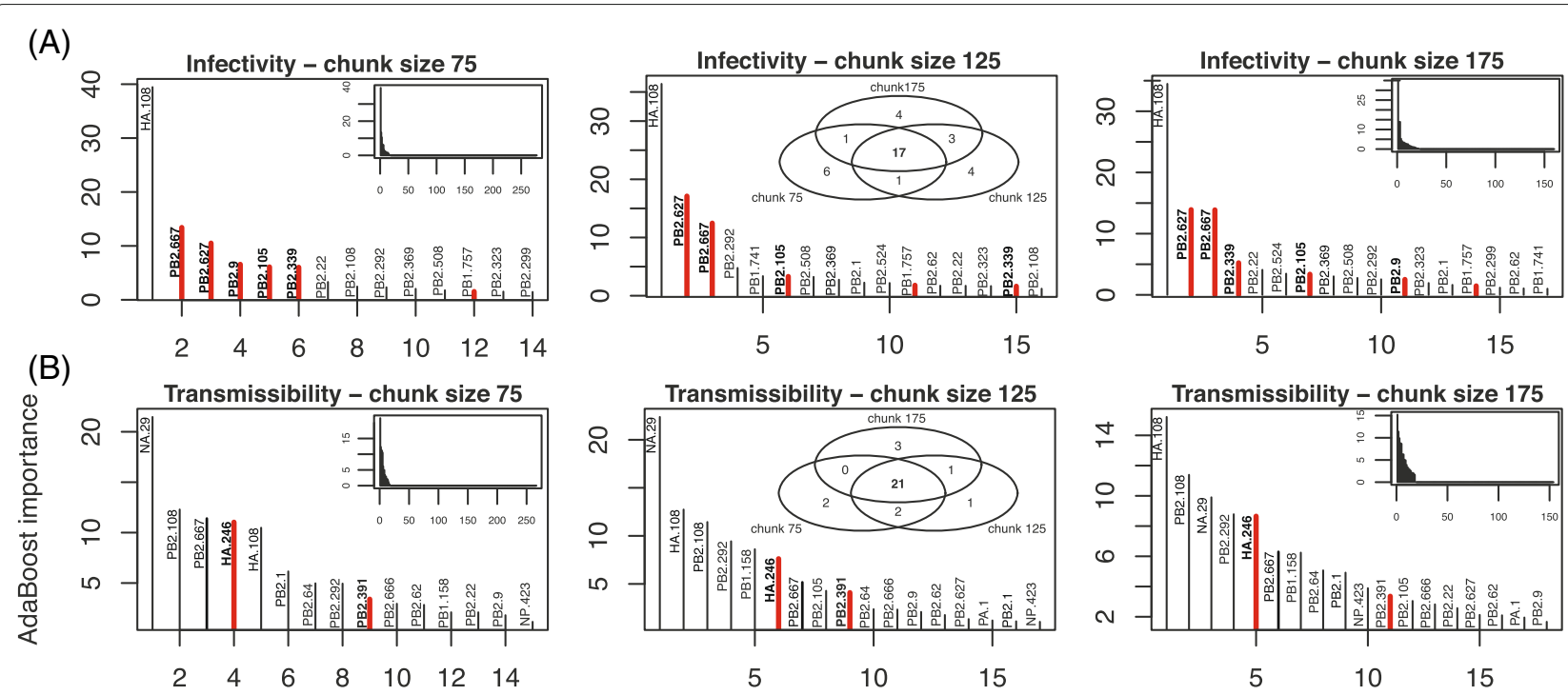

(C)
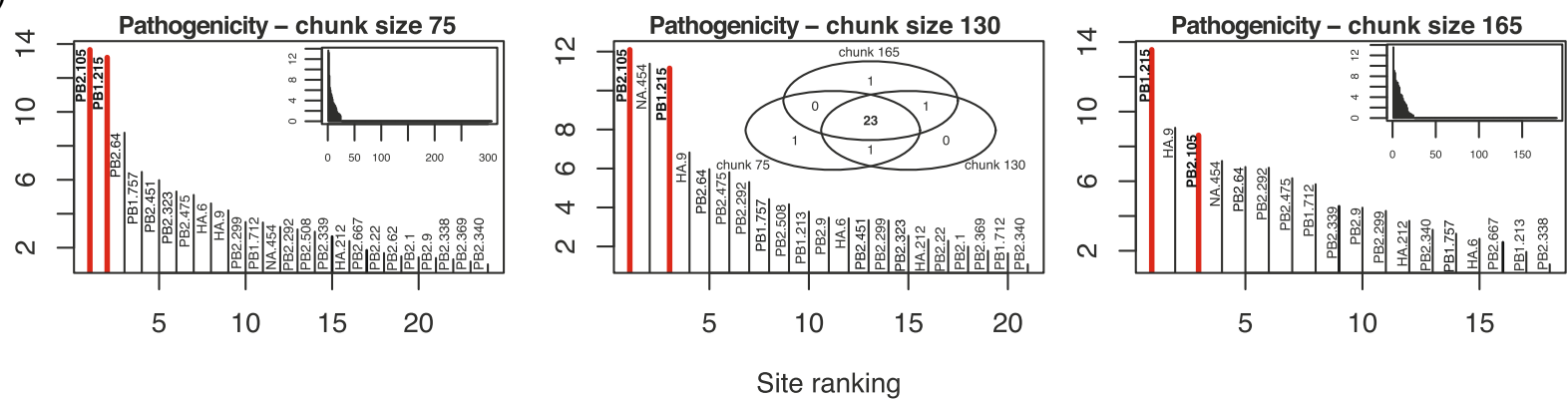

Fig. 2 Effect of chunk size on the distribution of importance of sites for the AB algorithm. The genes and sites identified as genetic determinants of influenza phenotypes are shown for: $\mathbf{a}$ infectivity, $\mathbf{b}$ transmissibility, and $\mathbf{c}$ pathogenicity. Only results for the smallest (75 amino acids), intermediate (125), and largest (175) chunk sizes are shown. Only the most important sites (importance $>1.5$ ) are shown in each panel, with sites backed by with experimental evidence highlighted in red. Insets show the whole distribution of importance values (left and right columns), and the Venn diagrams of the most important sites at all three chunk size (middle column)

One tendency that emerged when increasing chunk sizes was a decrease in sensitivity for the first pass of the algorithm, and perhaps an increase in its final specificity for both $A B$ and RRF. In the case of infectivity for instance, the algorithms found a total of about 275 sites at chunk size 75, and roughly 150 sites at chunk size 175 (Fig. 2, insets with complete distributions) for $\mathrm{AB}$, and 130 sites with RRF (Fig. 3, likewise). The same pattern was observed for the two other phenotypes, transmissibility and pathogenicity. Although beyond the scope of this work, it is possible that an optimal chunk size exists in terms of area under the Receiver Operating Characteristic curve (balance between sensitivity and specificity); however, larger labeled data sets would be required for testing this possibility.

\section{Rediscovery of influenza determinants}

So far the results showed that the predictions made by $A B$ were less stable across chunk sizes than those from
RRF, but they did not tell us anything about their accuracy. While the results in Figs. 2 and 3 suggested that more true positive sites were found by $A B$ (with an average across chunk sizes of 5.7, 2, and 2 for each phenotype) than by RRF (with similar averages at 3.7, 1.3, and 4.3), Table 1 suggests a more nuanced view, where RRF found $44 \%$ more sites than $A B$ that are backed by experimental evidence (thirteen with RRF, nine with $A B$ ). Note finally that $A B$ seemed to be better at finding sites in PB2 (the longer gene), while RRF's rediscoveries were more evenly spread. Unfortunately, with such small numbers, the significance of these results is difficult to gauge.

Focusing on each phenotype, infectivity had the largest number of sites detected, the largest number of experimentally validated positions, and was the phenotype with the most stable predictions among the three phenotypes - see Table 1 for a full list. However, a number of sites detected by our approach, sometimes with consistent high importance values (e.g., HA 108 for infectivity; NA 29 

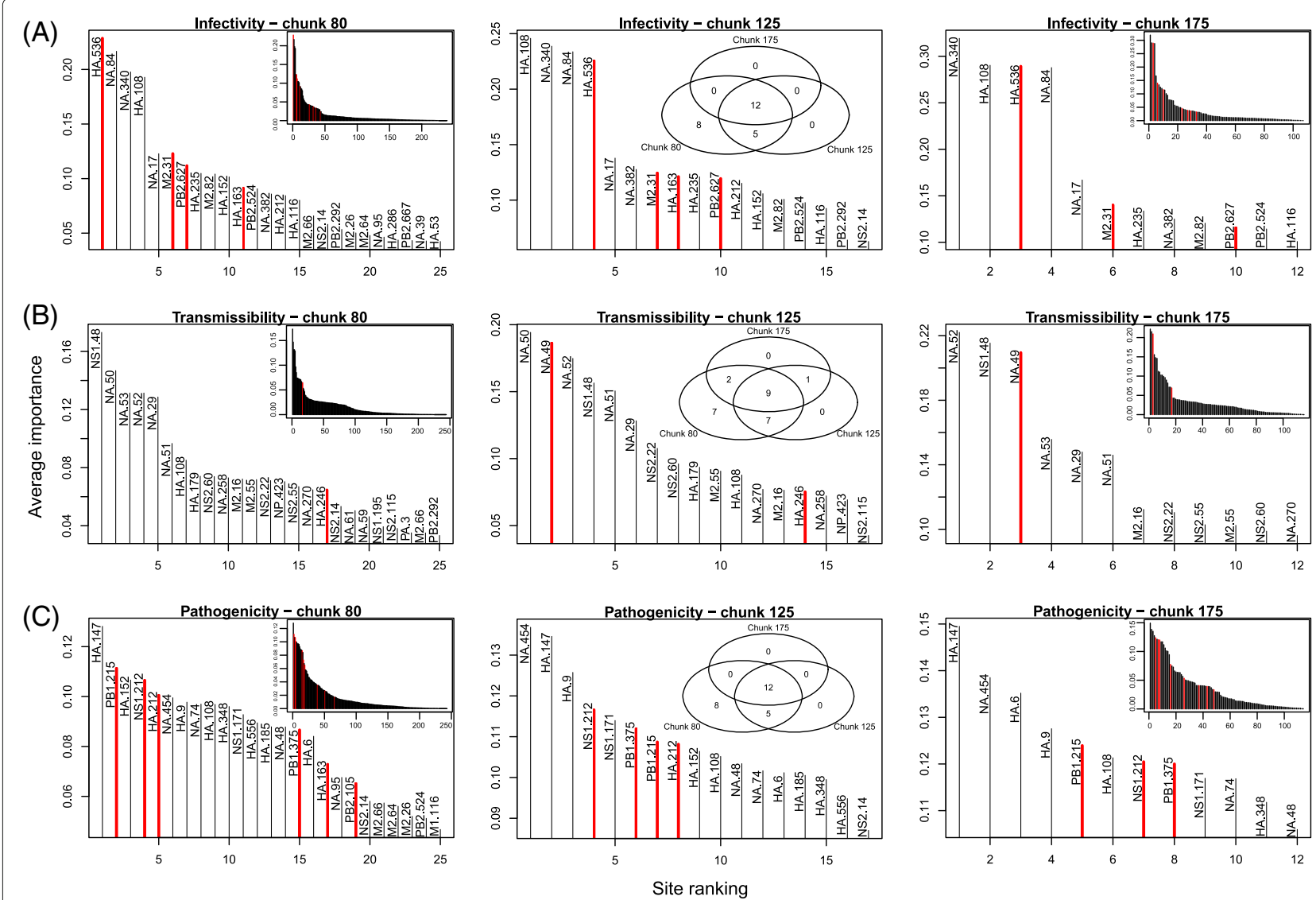

Fig. 3 Effect of chunk size on the distribution of importance of sites for the RRF algorithm. The genes and sites identified as genetic determinants of influenza phenotypes are shown for: $\mathbf{a}$ infectivity, $\mathbf{b}$ transmissibility, and $\mathbf{c}$ pathogenicity. Only results for the smallest ( 80 amino acids), intermediate (125), and largest (175) chunk sizes are shown. Only the most important sites (Gini index in top $90^{\text {th }}$ percentile of its distribution over all the sites) are shown in each panel, with sites backed by with experimental evidence highlighted in red. Insets show the whole distribution of importance values (left and right columns), and the Venn diagrams of the most important sites at all three chunk size (middle column)

for transmissibility), are, to our knowledge, not supported by any experimental evidence. As a result, it is possible that these predictions are false positives, even if absence of experimental evidence is not evidence of absence. We noted that with this small data set, cross-validation could not be performed to gauge the validity of these results: by splitting the samples ten times (equivalent to a leave-oneout resampling strategy), class imbalance increased dramatically, and the probability of drawing monomorphic alignment chunks (which lead the algorithm to fail) also increased. On the other hand, a number of key sites listed in the Influenza Research Database were also missed by our machine learning algorithms (Table 1). Furthermore, additional sites, which were not detected here, are known genetic determinants, but in other species. For instance, PB2 256 is known to increase polymerase activity, and hence boost infectivity, at least in pigs [46]. Likewise, PB2 $28,274,526$, and 607 do the same, but in birds [47]. As our alignment essentially contains sequences isolated from humans, it is possible that some of the sites we uncover are highly specific to this particular host. However, among these last five positions in PB2, only 526 was found to be polymorphic. Our results are therefore promising in that most of the known influenza sites (16 out of 20 , or $80 \%$ ), i.e. those supported by experimental evidence, were rediscovered by our algorithms.

\section{High sensitivity of RRF with chunking}

In order to better understand the performance of the RRF algorithm, we conducted a simulation study (a similar study for $A B$ could not be performed because of its high computational cost). For this, we generated sequence alignments on a 20-letter alphabet, representing the 20 amino acids found in the influenza proteome, with a single site whose amino acids match perfectly a binary phenotype (Additional file 1: Figure S13). We first generated alignments under a balanced design, where half of the sequences were from the first phenotype, just as in the influenza data. The results show that for alignments with at least 30 sequences, irrespective of their 
sequence lengths, RRF has maximum sensitivity (Fig. 4a), and high specificity (Fig. 4b). With data sets containing 20 sequences or fewer, sensitivity decreases quickly as sequence length increases, but as suggested by the empirical results, smaller chunk sizes can maintain sensitivity to at least $50 \%$ at 5000 sites. With ten sequences 5000 sites in length, as in our influenza data set, sensitivity is pretty much 0 , even for the smallest chunk size tested in our simulations (10\%). Additional simulations showed that, at a chunk size of $2 \%$, as in the influenza analysis above, sensitivity could reach $10 \%$, but also that doubling the number of sequences in the alignment could increase sensitivity to 80\% (Additional file 1: Figure S14). As expected, class imbalance decreased both sensitivity and specificity (Fig. 4c-d), which justifies why we tried to achieve a balanced distribution of phenotypes.

With such a balanced distribution, the specificity of RF was always larger than that of RRF (Additional file 1: Figure S15). However, sensitivity of RRF was higher than that of RF (Fig. 4e), and could reach $100 \%$ with as few as 20 sequences if DNA data were analyzed (Fig. 4i-j), even in the presence of class imbalance (Fig. 4k-l). In the balanced case, the difference between RF and RRF was highly significant $(P \ll 0.01)$, except for chunk sizes $\geq 40 \%$ (Additional file 1: Figure S16). Again, there was a very significant interaction between chunk size and the number of sequences, with sensitivity increasing with smaller chunk sizes and larger numbers of sequences, reaching 100\% with as few as 30 sequences and chunk sizes as large as 10\% (Additional file 1: Figure S16).

Altogether, our simulations are in line with the empirical results obtained on the influenza data containing few sequences, in that our true positive rates were low between 4 and 25\% (Fig. 3), but that a small increase in the number of sequences would significantly boost performances (Additional file 1: Figures S14-16).

\section{Unimpressive performance of $A B$ on $P$. aeruginosa}

Given these encouraging simulation and empirical results on the influenza data, for which experimental evidence supported some of the identified genetic determinants of three complex phenotypes (infectivity, transmissibility, and pathogenicity) with a small number of strains $(n=$ $10)$, we analyzed an alignment of previously sequenced bacteria $(n=26)$, for which we had access to minimum inhibitory concentration (MIC) values for three antibiotics (Ciprofloxacin, Ceftazidime, and Gentamicin) [25] and for which simulations suggested that we could reach a sensitivity $>80 \%$ with a specificity $\sim 100 \%$ (Fig. 4 ). We first employed $A B$, as this algorithm performed well in a recent small sample size application [16]. For each phenotype (MIC value; Fig. 5, top row), we ran the $A B$

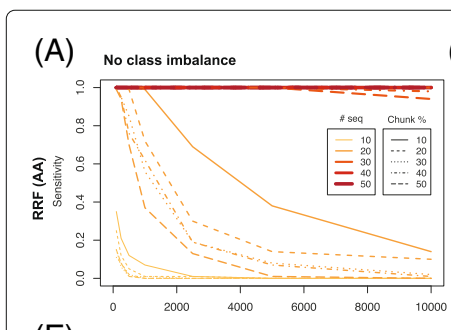

(E)

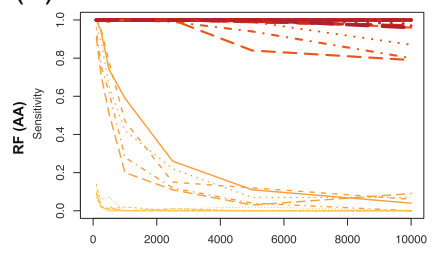

(I)

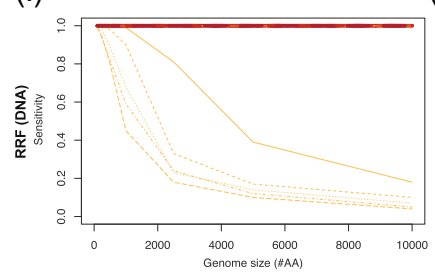

(B)

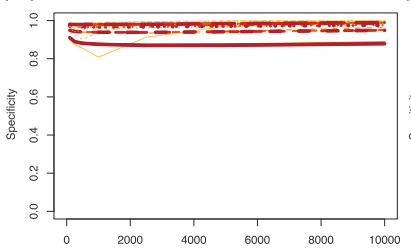

(F)

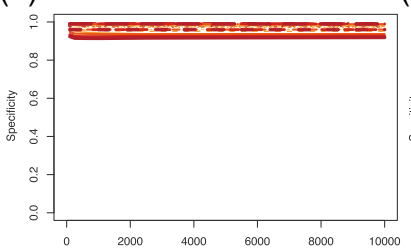

(J)

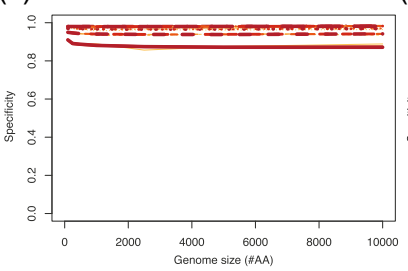

(C)

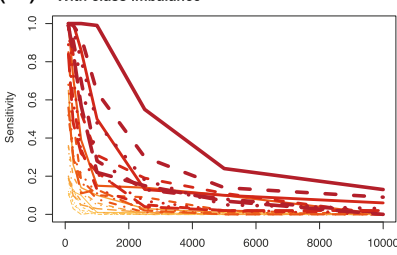

(G)

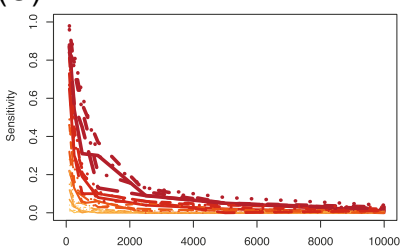

(K)

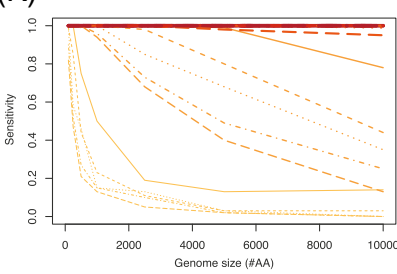

(D)

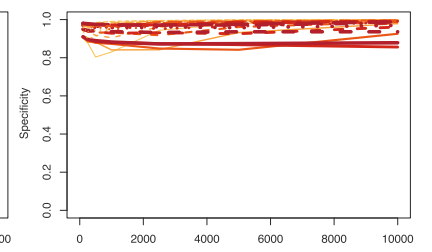

(H)

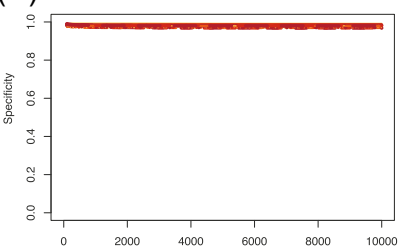

(L)

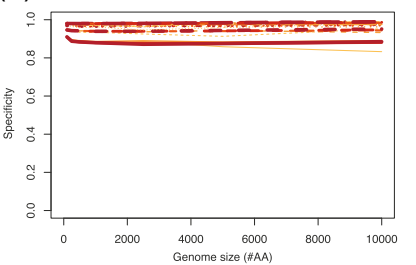

Fig. 4 Impact of chunking and data size on sensitivity and specificity. Simulations were conducted to assess the impact of chunking, number of sequences and length of protein alignments on (a) sensitivity and (b) specificity of the RRF algorithm, with no class imbalance, or in the presence of class imbalance $(\mathbf{c})$ and $(\mathbf{d})$, respectively. Similar simulations were conducted under the RF algorithm $(\mathbf{e}),(\mathbf{f}),(\mathbf{g})$ and $(\mathbf{h})$, respectively, still for protein data, and under RRF for DNA data (i), (j), ( (k) and (I), respectively 


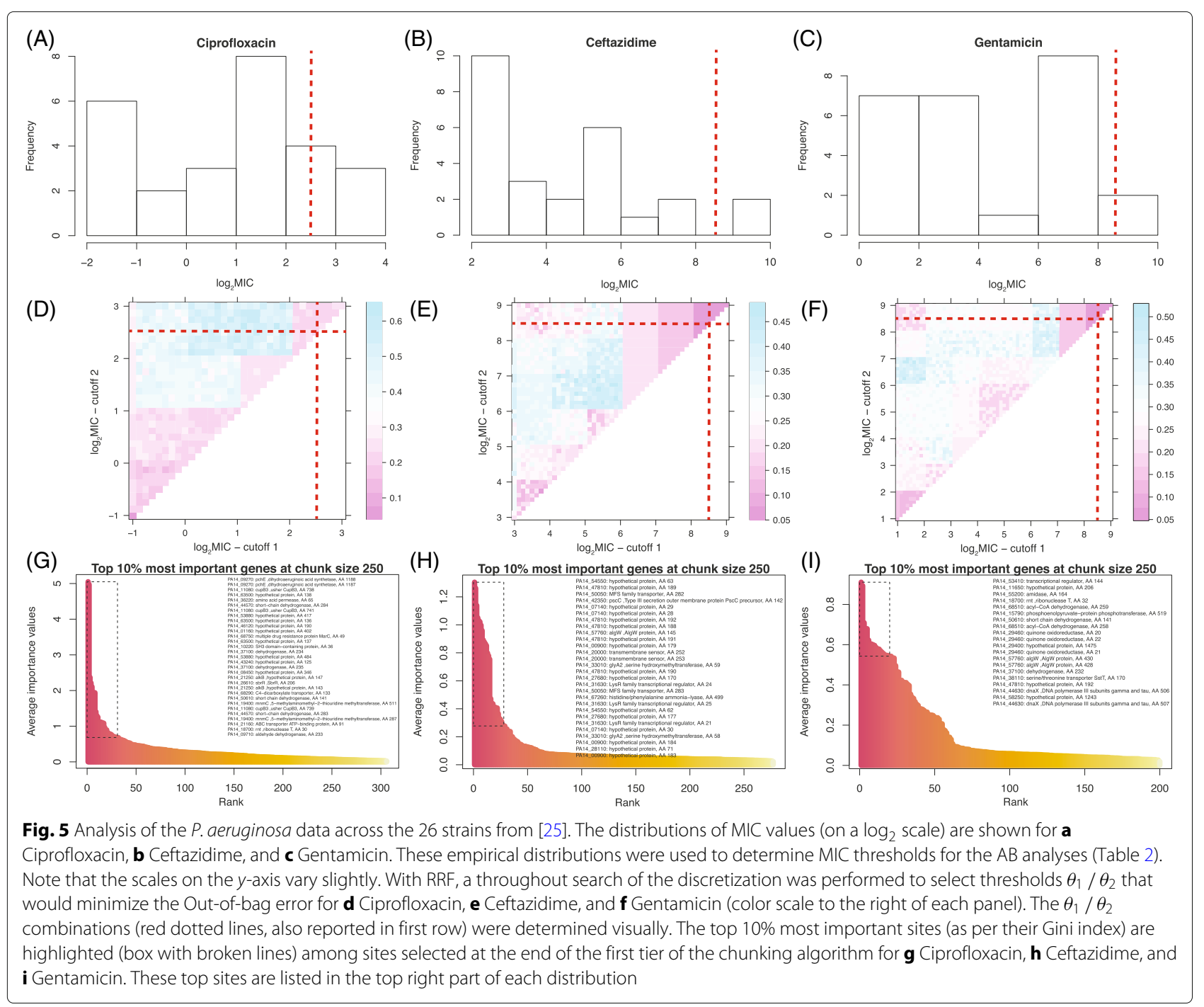

algorithm twice with a chunk size of 5000 ("runs A"; 1\% of total alignment length), and twice with a chunk size of 1000 ("runs B"; 0.2\% of total alignment length). This allowed us to further test how chuck size affects which sites are detected, and how stable this detection is across different chunk sizes. As with the influenza alignment, increasing chunk size tended to lower sensitivity in the first pass of the algorithm, as fewer positions of interest had high importance (Additional file 1: Figure S17, insets). On the other hand, sensitivity seemed to be restored, in larger chunk sizes, after the second pass, as more sites with importance values $>1$ were found for all phenotypes (Additional file 1: Figure S17, main panels). Additional file 1: Figure S18 shows that only the most important sites were identified with very similar importance values across the four runs (and hence the two chunk sizes). When only the top 25 sites were compared across these four runs, only five to seven sites were shared (Additional file 1:
Figure S19). Some of these predictions are sensible: DNA gyrase subunit $B$ (gyrB) is known to be involved in drug resistance [48, 49], hypothetical protein PA14_40040 (see Ceftazidime phenotype runs) is known for its involvement in antibiotic biosynthesis processes [50], and tonB2 is inferred to be an iron transporter which potentially affects bacterial drug resistance [51]. Detecting genes that were previously unknown to be involved in drug resistance is not uncommon, as a recent study of antibiotic resistant P. aeruginosa found that $12 \%$ of the assayed strains carried novel genetic determinants [52]. What is more unexpected though is that some of these genes are not supposed to be involved in the mode of action of these specific drug: for instance, Ciprofloxacin, as a fluoroquinolone, is known to disrupt gyrB [49], but it is also the only drug in our results for which gyrB was not identified (Table 3). Likewise, sbrR is a factor involved in swimming ability [53], and would hence had been expected to be 
involved in the resistance to cephalosporins such as Ceftazidime, not the aminoglycoside Gentamicin (Table 3).

All these results were obtained with $A B$ under one way of discretizing the MIC distributions (setting 1; Table 2). By using two other discretization schemes of the MIC distributions, it is striking that there was almost no consistency among the results (Table 3). The only proteins identified under all three settings were PA14_40040, for Ceftazidime, and tonB2 for Gentamicin. Even well-known factors such as gyrB were not identified under any of the three settings. Potential nonexclusive reasons for this lack of consistency include class imbalance and a rather small number of strains $(n=26)$ leading to unstable training.

To better quantify general performance of $A B$ in the case of P.aeruginosa, we finally performed a ten-fold cross-validation $(\mathrm{CV})$ analysis on the 26 strains. The confusion matrix, which depicts the predicted number of strains in each MIC category (low / medium / high) in rows, and observed numbers in columns, showed that under setting 1, class imbalance can be quite high for resistance to the three drugs, systematically leading to one of the three MIC categories with absolutely no prediction. This can be seen for instance at medium MIC values for Ceftazidime and Gentamicin (middle row):

$$
\begin{gathered}
\text { Ciprofloxacin: }\left[\begin{array}{lll}
0 & 0 & 0 \\
0 & 3 & 3 \\
2 & 6 & 12
\end{array}\right] \text { Ceftazidime : }\left[\begin{array}{lll}
9 & 1 & 1 \\
0 & 0 & 0 \\
1 & 4 & 9
\end{array}\right] \\
\text { Gentamicin: }\left[\begin{array}{lll}
9 & 1 & 2 \\
0 & 0 & 0 \\
5 & 1 & 10
\end{array}\right]
\end{gathered}
$$

The resulting error rates for predicting the correct MIC categories were $42.31 \%, 30.77 \%$, and $26.92 \%$, respectively. As there are three discrete MIC categories, with little class imbalance, the error rate for a random classification should be close to $67 \%$. The $\mathrm{AB}$ algorithm did not have a good performance, even if it still did better than chance alone at predicting the MIC category (low / high) of an unknown bacterial strain from its proteome only. Yet, these performances explained neither the instability of the pseudomonas results, nor their lack of complete biological sensibility.

Table 2 The different sets of MIC thresholds employed to assess the robustness of the classification results with $A B$ in the case of $P$. aeruginosa

\begin{tabular}{llll}
\hline Drug & Setting 1 & Setting 2 & Setting 3 \\
\hline Ciprofloxacin & $-1 / 1.5$ & $0 / 2$ & $-1 / 2$ \\
Ceftazidime & $4 / 6$ & $6 / 8$ & $4 / 8$ \\
Gentamicin & $4 / 6$ & $6 / 8$ & $4 / 8$
\end{tabular}

Shown are the thresholds $\theta_{1} / \theta_{2}$ used on a $\log _{2}$ MIC scale: for instance, setting 1 for Ciprofloxacin means that MIC is low when $\leq-1\left(\theta_{1}=-1\right)$, high when MIC $>1.5$

$\left(\theta_{2}=1.5\right)$, and medium in-between
One possibility is that $A B$ was actually overfitting the data: this happens when a particular classifier accommodates all the most minute singularities of a train data set, and performs poorly on a test data set. While $A B$ is generally considered to be robust to overfitting $[15,54]$, this can occur when too many iterations are performed [19] - which prompted some authors to consider $m_{\text {final }}$ as the AB's main tuning parameter [55]. To assess whether overfitting was responsible for the poor pseudomonas results, we reran the $\mathrm{AB}$ analyses under setting 1 (Table 2$)$ with different numbers of iterations $\left(m_{\text {final }} \in\right.$ $\{10,25,50,100,200\})$, both in the first and second passes of the chunking algorithm. To further assess the potential interaction with chunking, these additional analyses were run for both chunk sizes (1000 and 5000). Additional file 1: Figure S20 shows that most analyses show a convex (concave up) CV error rate, which entails the existence of an optimal $m_{\text {final }}$, that allowed optimal errors rates to be as low as $25 \%$. Different chunk sizes had different optimal $m_{\text {final }}$ : for Ciprofloxacin, e.g., the minimum CV error rate was at $m_{\text {final }}=50$ at chunk size of 5000, but at the edge of the $m_{\text {final }}$ interval tested for chunk size 1000. Importantly, under both chunk sizes, the only unambiguously identified protein was trpI (PA14_00460, Table 3, underlined), a transcriptional activator implicated in Tryptophan biosynthesis. Mutations in this gene lead to reduced (albeit modest) swimming motility [56], implicated in ciprofloxacin resistance [57]. Table 3 shows that for the two other drugs, under optimal $m_{\text {final }}$ values, the genes identified with both chunk sizes were already among the top ten genes identified by the previous analysis, which suggests that, for a given discretization scheme of the MIC values (phenotypes), these gene lists were fairly robust to the number of iterations ( $\left.m_{\text {final }}\right)$, and that overfitting was probably not an issue in this application.

\section{RRF outperforms $A B$ on $P$. aeruginosa}

$A B$ showed some obvious shortcomings when analyzing the pseudomonas data, some of which could be linked to our discretization of the MIC curves, in addition to large memory footprints and runtimes. RRF allowed us to address all these issues: as this algorithm ran almost four orders of magnitude faster than $A B$ (Fig. 1), it was possible to perform a more thorough search of the discretization space by varying systematically the position of the two thresholds $\left(\theta_{1}, \theta_{2}\right)$ (Fig. 5a-c). The pattern of Out-ofbag error, with triangles of low errors along the diagonal (Fig. 5d-f), showed that these data should be analyzed with a single MIC threshold: dissimilar $\left(\theta_{1}, \theta_{2}\right)$ thresholds led to high errors; similar $\left(\theta_{1}, \theta_{2}\right)$ thresholds led to low errors. This pattern was not unexpected given the coarseness of the MIC distributions (Fig. 5a-c). While both Ceftazidime and Gentamicin had clear threshold minimizing the Outof-bag error, multiple choices existed for Ciprofloxacin. 
Table 3 Gene lists of the most important candidates for drug resistance in P. aeruginosa

\begin{tabular}{llll}
\hline Drug & Setting 1 & Setting 2 & Setting 3 \\
\hline Ciprofloxacin & trpl, transcriptional regulator Trpl & hypothetical protein & trpl, transcriptional regulator Trpl \\
\cline { 2 - 3 } & lysin domain-containing protein & HIS/PHE ammonia-lyase & tag, DNA-3-methylad. glycosidase I \\
& tag, DNA-3-methylad. glycosidase I & LysR family transcriptional regulator & lysin domain-containing protein \\
& recF, recombination protein F & & glutamine synthetase \\
& D,D-heptose 1,7-bisphos. phosphatase & & hypothetical protein
\end{tabular}

Ceftazidime hemolysin activ./secret.prot

hypothetical protein (PA_40040)

gyrB, DNA gyrase subunit $B$

\author{
hypothetical protein (PA_40040) \\ sensor/response regulator hybrid
}

hemolysin activ./secret. prot

hypothetical protein (PA_40040)

gyrB, DNA gyrase subunit $B$

hemagglutinin

recQ, ATP-depend. DNA helicase

tonB2, hypothetical protein

hemagglutinin

sbrR, SbrR

hemolysin activ./secret. prot

\section{tonB2, hypothetical protein}

hemagglutinin

nirN, c-type cytochrome

\section{tonB2, hypothetical protein hemagglutinin \\ tufA, elongation factor Tu \\ hemolysin activ./secret. prot}

Shown are the genes identified in all four runs under the four settings defined in Table 2. For each drug, the genes identified in all three settings are highlighted (boldface), as well as those found in two out of the three settings (italics). Gene names that are underlined (setting 1 only) are those identified during the cross-validation experiment, under both chunk sizes

To identify genetic determinants at high drug levels (the mutations conferring the highest levels of resistance), a high threshold was chosen (Fig. 5a, d).

Figure $5 \mathrm{~g}$-i shows the most important sites identified by RRF. To determine which of these sites are potential candidates for drug resistance, we used the empirical rules derived from the analysis of influenza data, as those analyses were informed by experimental evidence, and validated by our simulations (Fig. 4). As above, we expected that most of the true positive sites are (i) found in at least $50 \%$ of the repeated parts of RRF, and (ii) in the top $10 \%$ of the global distribution of importance (Gini) values. For influenza, these two rules allowed us to capture all but one of the experimentally-validated sites, while minimizing the number of false positives (see Additional file 1: Figure S19). Under these two rules of thumb for site discovery, we found lists of sites (Fig. 5) that are completely different from those found with $\mathrm{AB}$ (Table 3). However, these lists of sites were very stable across a wide range of chunk sizes (Additional file 1: Figure S21), and their content made sense in light of what is known about these three drugs, which are commonly employed to treat $P$. aeruginosa infections of cystic fibrosis patients (Table 4). Note that Out-of-bag errors rates were all $<10 \%$ (Fig. 5), when CV error rates under $\mathrm{AB}$ were at least $25 \%$. These lists of candidate genetic determinants for drug resistance are still long, with many hypothetical proteins, and hence remain problematic for experimental validation, but large and deep mutational screens have already started to revolutionize the field [70].

Lastly, to evaluate the overall performance of RRF on the pseudomonas data set, we conducted additional simulations, as above, but with a smaller chunk size $(0.2 \%$, as in "runs B"), varying the number of sequences between 24-30, and increasing sequence lengths all the way to $10^{6}$ sites. Only 25 replicates were done in these conditions, but these results suggest that we would need at least 30 pseudomonas proteomes (of length 500k sites) to reach a sensitivity of $90 \%$, and a specificity just above $60 \%$ (Additional file 1: Figure S22). With the data set that we had access to (26 proteomes), sensitivity was $<15 \%$. Larger proteomes, containing a million polymorphic sites or more, will require sequencing at least 30 individuals to reach sensitivity values of at least $80 \%$.

\section{Conclusions}

In order to find the genetic determinants of particular phenotypes whole genome or proteome data, we implemented and tested two machine learning algorithms based on adaptive boosting, and random forests. Our use of these machine learning algorithms can be characterized as unbiased, in that they gauge the importance of every 
Table 4 Characterization of RRF drug resistance candidates in the pseudomonas data from the literature

\begin{tabular}{|c|c|c|c|}
\hline Drug & Gene & Evidence & References \\
\hline \multirow[t]{9}{*}{ Ciprofloxacin } & \multirow[t]{2}{*}{ pchE } & $\begin{array}{l}\text { Siderophore family, extracellular iron-acquisition system, upregulation associated with } \\
\text { exposure to natural quinolones }\end{array}$ & [58] \\
\hline & & $\begin{array}{l}\text { Iron is required for virulence and is deficient in the human lung environment of these } \\
\text { clinical strains }\end{array}$ & [59] \\
\hline & cupB3 & $\begin{array}{l}\text { Part of an outer membrane porin family, mutation that reduce membrane permeability } \\
\text { are linked to Gram-negative bacterial mechanisms for antibiotic resistance }\end{array}$ & [60] \\
\hline & permease & $\begin{array}{l}\text { Reduces accumulation of drug inside cell by decreased cell wall permeability or by } \\
\text { pumping drug out }\end{array}$ & [61] \\
\hline & ABC transporter & See permease & {$[61]$} \\
\hline & $\mathrm{SH} 3$ & $\begin{array}{l}\text { Controls numerous protein-protein interactions, some implicated in virulence of } \\
\text { pathogenic bacteria }\end{array}$ & [62] \\
\hline & alkB & $\begin{array}{l}\text { DNA repair system (fluoroquinolones prevent proper winding and unwinding of DNA dur- } \\
\text { ing replication), also affects outer membrane lipids - and thus permeability - it may affect } \\
\text { antibiotic resistance }\end{array}$ & {$[60]$} \\
\hline & sbrR & Anti-sigma factor, identified as necessary during the chronic infection of respiratory tracts & [53] \\
\hline & $\mathrm{mnmC}$ & $\begin{array}{l}\text { Part of tRNA modification, and thus protein synthesis, no obvious antibiotic or lung } \\
\text { environment connection }\end{array}$ & \\
\hline \multirow[t]{5}{*}{ Ceftazidime } & MFS & $\begin{array}{l}\text { Membrane translocases, include many multidrug resistant proteins of Gram-positive } \\
\text { bacteria }\end{array}$ & [63] \\
\hline & $\mathrm{pscC}$ & Type III secretion outer membrane protein, probable general resistance candidate & {$[60,64]$} \\
\hline & algW & Mutations are known to confer susceptibility to the beta-lactam family of antibiotics & {$[65]$} \\
\hline & glyA2 & $\begin{array}{l}\text { Produces anti-oxidant coenzymes, involved in cell response to TiO2-based nanocompos- } \\
\text { ite antimicrobials }\end{array}$ & {$[66]$} \\
\hline & lysR & $\begin{array}{l}\text { Associated with minimum inhibitory concentration of antibiotics and oxidative stress } \\
\text { chemicals }\end{array}$ & {$[67,68]$} \\
\hline \multirow[t]{3}{*}{ Gentamicin } & rnt & This ribonuclease would have a logical role in degrading 305 bound by the antibiotic & \\
\hline & algW & Correlated to Ceftazidime resistance? & \\
\hline & quinone OR & $\begin{array}{l}\text { Antibiotic resistance in response to antibiotics that inhibit protein synthesis - including } \\
\text { binding of the } 50 \text { ribosomal subunit }\end{array}$ & [69] \\
\hline
\end{tabular}

single position in a proteome without any a priori assumptions - in the same way as are often characterized GWAS [4] or RNA-seq studies [71-73]. However, because each proteome contains a very large number of positions, these algorithms could not be run 'as is' on the entire alignment, even after removing invariant positions. In regulatory genomics, where the objective is to uncover splice junctions, such machine learning algorithms typically focus on sequence windows centered on the traits of interest, thereby reducing the feature space $[74,75]$. Here, we did not consider such prior knowledge (when it existed) to pre-define features, and instead took a more agnostic approach, focusing on the entire alignment of polymorphic positions. The resulting computational burden prompted us to develop the chunking algorithm, especially in the case of $A B$, where the initial alignment is chopped up into smaller parts or chunks. Each algorithm, $A B$ and RRF, is run in a first pass on each chunk, to determine a set of positions of interest, which are then collated for a second pass to rank these positions by their importance in predicting a particular phenotype. Here we showed that chunking improves runtimes, without qualitatively affecting performance, both in terms of which positions are identified, and their importance values and may even increase sensitivity of model predictions. Although our RRF algorithm is based on random forests, it is not equivalent to this latter algorithm run on more trees because we combine the results of each repeat by taking their consensus. RRF may also be reminiscent of the iterated RF algorithm (iRF) [76], but while RRF is less sophisticated, the stability of model predictions are definitely improved.

We then showed by analyzing a data set in which the genetic determinants of complex phenotypes are known (influenza) that both $\mathrm{AB}$ and RRF correctly identified some positions supported by experimental evidence [77], but that the top results also included some potential false positives, and missed some known sites. Simulations suggested that excellent performance could be obtained, with sensitivity and specificity both close to $100 \%$, but with larger data sets ( $>20$ sequences). The analysis of a second and larger data set (26 sequences), 
for which the genetic determinants of complex phenotypes are not so well known (pseudomonas) allowed us to identify positions in genes known, or predicted to be, involved in the phenotypes assayed, with more sensible results obtained with RRF than with $\mathrm{AB}$. Altogether, predictions based on machine learning algorithms can allow for a quicker discovery process of the genetic determinants of complex phenotypes, but should be more thoroughly compared with traditional GWAS approaches. Far from being restricted to finding resistance genes and mutations, our approach is amenable to predicting any kind of phenotype/genotype relationships, including disease-causing mutations. However, such a use of machine learning to determine phenotype/genotype relationships might only blossom when both phenotyping and genome sequencing costs become low enough to perform these analyses on many more individuals.

\section{Methods}

\section{The influenza data}

The complete proteome of $n=10$ Influenza A strains, containing the twelve canonical genes usually found in these viruses [18], were retrieved from the Influenza Research Database [24] using their search tool based on phenotype characteristics. The retrieved strains included all viral samples with experimental evidence supporting an increase of infectivity, transmissibility, and pathogenicity, the three main phenotypes in this database (retrieved Sep 2016). The detection of a polybasic cleavage site was used as a proxy for pathogenicity; while the presence of such a site alone does not indicate an increase of pathogenicity, it is nonetheless present in highly pathogenic strains [78]. The phenotypes were encoded as binary variables, since phenotypic data were only available as a Yes / No statement. Our analyses were performed blindly, as no indication of any particular mutation was included in the strain name (Table 5). Note that our selection of strains tried to minimize class imbalance, so that both infectivity and pathogenicity have a 1:1 ratio, while transmissibility data are a bit more uneven (3:7; Table 5).

The corresponding proteomic data were downloaded from the Influenza Virus Resource [79]. We focused on amino acid data, assuming that phenotypic differences are caused by nonsynonymous mutations. Only the ten most common proteins found in all influenza strains (PB2, PB1, PA, HA, NP, NA, M1, M2, NS1, and NS2) were retrieved. This was done to ensure that the results obtained could be applied to the widest selection of strains possible. The selected viral strains were essentially from human hosts. This was done to prevent any potentially confounding factors from arising due to the different cellular targets [80], or due to the large sequence difference between avian and mammalian subtypes [81]. Strains containing only one
Table 5 List of the Influenza A strains and their associated phenotypes, as used in the training of the machine learning algorithms

\begin{tabular}{llll}
\hline Strain name & Infectivity & Transmissibility & Pathogenicity \\
\hline A/HongKong/156/97 & No & Yes & Yes \\
A/HongKong/213/2003 & No & Yes & Yes \\
A/Indonesia/5/2005 & No & No & No \\
A/Indonesia/7/2005 & No & No & No \\
A/PuertoRico/8/34 & Yes & No & No \\
A/Swine/Indiana/1726/1988 & Yes & Yes & No \\
A/Turkey/15/2006 & No & No & No \\
A/VietNam/1203/2004 & Yes & No & Yes \\
A/VietNam/3046/2004 & Yes & No & Yes \\
ANietNam/3062/2004 & Yes & No & Yes \\
\hline
\end{tabular}

For pathogenicity, polybasic cleavage was used as a proxy

segment (e.g., PA/Fort Monmouth/1/47-MA(H1N1)) were discarded.

The segments of each strain were individually aligned with MUSCLE 3.8.31 [82] to ensure accuracy, and were then concatenated into a single alignment. Any missing segment in any strain was replaced with a row of gap characters to (i) ensure proper concatenation, and (ii) prevent a mismatching of protein segments between the different influenza strains, and thus prevent distortion of the alignments. After the segment concatenation, invariant sites were removed from the alignment. This was done to reduce the computational time required for adaptive boosting, as sites without mutations do not contain any information relevant to the analysis.

\section{Simulations}

Protein alignments were simulated based on a 20-letter alphabet by drawing amino acids with replacement from a uniform distribution. Only one site was perfectly associated with a binary phenotype. Simulations could be balanced, where each phenotype is in a 1:1 ratio, or unbalanced, where only two sequences are from the first phenotype. Number of sequences were taken in $\{10,20,30$, $40,50\}$, the length of each alignment took values in $\{100$, $250,500,1000,2500,5000,10,000\}$, and the chunk size changed from 10 to $50 \%$ of the total alignment length in $10 \%$ increments. One hundred replicates were performed under each condition. Sensitivity and specificity of each simulation condition were recorded, both under RRF, and RF for comparison purposes. To evaluate the impact of the size of the alphabet on performance, another round of simulations were performed on a 4-letter alphabet (RRF only), representing DNA data. These simulations allowed us to count True Positive (TP), False Negative (FN), True 
Negative (TN) and False Positive results, and deduce sensitivity $(\mathrm{TP} /(\mathrm{TP}+\mathrm{FN}))$ and specificity $(\mathrm{TN} /(\mathrm{TN}+\mathrm{FP}))$ from these.

\section{The pseudomonas data}

The proteome alignment of P. aeruginosa was generated by concatenating the coding regions of the $n=26$ P. aeruginosa genomes previously published [25]. With a reference database of PA14 (http://www.pseudomonas.com; [83]), an alignment for each open reading frame (ORF) was created using an in-house pipeline that: (i) stored BLASTn 2.2.30 [84] results for each ORF of each non-PA14 genome, (ii) discarded any results with identity $<90 \%$, (iii) assembled alignments for each ORF ensuring a genome's sequence was used only once. This was achieved by first building a scaffold from genomic sequence with only one BLASTn result, then extending incomplete scaffolds, i.e., those that did not cover the full range of their respective PA14 reference sequence. Extensions were done using BLASTn results that did not correlate with higher percent identity to another incomplete scaffold nor overlapped the current scaffold by more than 30 nucleotides. Scoring of genomic ranges and overlaps was performed using Bioconductor's function GRanges [85]). Following scaffold assembly, (iv) sequences were aligned using MUSCLE 3.8.31 [82]. Any aligned ORF with $<50 \%$ of strains having non-gap characters in at least $90 \%$ of reference sites (established via PA14 sequence) were discarded. Lastly, the remaining aligned ORFs were concatenated with the perl script catfasta2phyml.pl (by Johan Nylander: https://github.com/nylander/catfasta2phyml/commit/ $5035 \mathrm{eb})$. This resulted in an alignment containing 5944 of the 5977 ORFs in the PA14 reference genome, and a total of $1,974,843$ amino acid positions. Gene annotations were obtained from the file UCBPP-PA14.CSV available at http://www.pseudomonas.com/downloads/ pseudomonas/pgd_r_18_1/Pseudomonas/complete/gtfcomplete.tar.gz.

Each of these 26 strains had previously been characterized phenotypically, with respect to their antibiotic resistance to three different drugs: Ciprofloxacin, Ceftazidime, and Gentamicin [25]. All three are broad-spectrum drugs, used to treat patients infected by P.aeruginosa, and all three belong to different families of antibiotics (Ciprofloxacin is a fluoroquinolone, Ceftazidime is a cephalosporin, and Gentamicin is an aminoglycoside). As such, each drug has a different mode of action: fluoroquinolones inhibit enzymes such as DNA gyrase and topoisomerase IV, involved in the replication of DNA [49]; cephalosporins interrupt the synthesis of the peptidoglycan layer forming the bacterial cell wall [86]; aminoglycosides bind to the 30s ribosomal subunit and inhibit protein synthesis [87]. Hence, different genes can be expected to be involved in the resistance to these antibiotics.
Resistance had been quantified by means of MIC assays, where a growth medium containing antibiotics is serially diluted, in two-fold steps, before an equal volume of overnight bacterial culture be inoculated into each dilution. After at least $16 \mathrm{~h}$ of growth in these conditions, the MIC is defined as the minimum antibiotic concentration that does not permit growth.

\section{Predictive modeling}

Two machine learning algorithms were used to construct a model to predict each phenotype from the proteomic information (proteome) in both data sets, influenza and pseudomonas. The first was $A B$ [12], as implemented in the $\mathrm{R}$ package adabag [88], while the second was the RF [17] algorithm from the $\mathrm{R}$ package randomForest 4.6-14 [89]. All scripts were run in R 3.5 [90], and are available from https://github.com/sarisbro, alongside the data used.

The features included in both machine learning algorithms were the same: the amino acids of each proteome alignment. To keep track of site identity, each alignment was stored as a matrix, where column names contained the name of each ORF and the amino acid position within each ORF. As only polymorphic positions in the alignments are potentially informative, invariant sites were first discarded. This left 4392 polymorphic positions in the influenza alignment, and 511,780 in the P.aeruginosa alignment.

While the $A B$ algorithm used was unaltered, with the total number of iterations left to its default value $\left(m_{\text {final }}=\right.$ number of sites in the alignment), we slightly modified the $\mathrm{RF}$ algorithm. Indeed, due to the stochastic nature of this algorithm, RF can lead to different rankings of the most important features across different runs of the same model on the same data. To alleviate this issue, we ran each random forest model ten times, and kept only sites that have a Gini index $>0.075$. These ten sets of features are then combined by (i) taking their consensus at a certain threshold, and (ii) keep only the top $10 \%$ consensus features (Additional file 1: Figure S1). A similar modification of $A B$ could have been attempted, but was not pursued here due to large memory footprint and runtimes of this algorithm.

Indeed, one limitation of most machine learning algorithms is that they can require a large amount of memory to run, especially in the case of data sets with large numbers of features, such as with the P. aeruginos $a$ alignment. To alleviate this issue, alignments were split into sequential chunks of pre-specified sizes, ranging from 75 to 175 amino acids (by increments of five) for the influenza data, and chunk sizes being either 1000 or 5000 (AB), or ranging from 80 to 4000 (RRF) amino acids for the pseudomonas data. Note that these splits are largely random as segments of the influenza genome were "randomly" concatenated (by convention, segments are ordered by 
decreasing length, just like chromosomes in Eukaryotes), and protein-coding genes in pseudomonas were "randomly" concatenated (based on their order in the PA14 strain). All the computing in this step can be parallelized, so that each classifier can be run on each chunk independently by distributing the analyses over eight threads with the R foreach package 1.4.4 [91]. The RRF algorithm was first run on each chunk during the first pass of the chunking algorithm, hereby producing a set of positions of interest. Only those with a Gini index $>0.075$ were kept (first pass of the chunking algorithm), collated, and the classifier was run a second time on these (i.e., during pass 2 of the chunking algorithm), to produce the final set of most important sites (i.e., predictors of each phenotype in the top $10 \%$ Gini indices in the second pass of the chunking algorithm).

The performance of the machine learning algorithms was assessed using ten-fold cross-validations (AB) and Out-of-bag errors (RRF). For cross-validations, the alignment was divided into ten sets of sequences (samples), nine sets being used for training and the remaining one for testing. That process was then repeated for all ten subsets [88]. In the context of our chunking procedure, cross-validation was performed on the second pass of the $A B$ algorithm. Out-of-bag errors were computed on the predictions based on the bootstrapped trees that were not included during training.

As the evolution of antimicrobial resistance in P. aeruginosa can include many understudied sites [92], only the influenza data have sufficient experimental validations to which we can compare our predictions and thus determine their accuracy. In order to learn from this data set how to best balance true positives (sites that are known to be experimentally validated and are detected) and false positives (sites that are detected but are not experimentally validated) from the distribution of their importance values, we defined two thresholds (Additional file 1: Figure S1). First, the expectation is that the most important sites will mostly have true positives, so the first threshold used the influenza data to determine what top percentile of ranked importance values maximizes the number of true positive. We refer to this threshold as the "percentile threshold." Then, to minimize the number of false positives among these top sites based on the repeated nature of the RRF, we implemented a second threshold, the "consensus threshold," which works as follows: all replicates of the RRF were run independently, and only sites that were predicted at a certain percentile threshold (i.e., a consensus) of all the runs were logged. Among these, only those that were found, say in $90 \%$ of the runs ( 9 runs out of 10), were considered as "genetic determinants." Intuitively, the higher this consensus threshold, the lower the number of false positives. We then varied both threshold on the influenza data to (i) maximize the number of true positives (percentile threshold) while (ii) minimizing the number of false positive (consensus threshold). We employed these thresholds to identify genetic determinants in the pseudomonas data.

Finally for the pseudomonas data, one additional step was required. As the algorithms used to identify the genetic determinants of phenotypes require categorical data, MIC distributions were discretized. To do so, and in the case of $A B$ first, the distribution of $\log _{2}$ MIC of each drug was first plotted, and appeared to be trimodal; hence, it seemed natural to design a classification with three categories: 'low', 'medium', and 'high' MIC. The boundaries between these categories were determined to minimize class imbalance, and hence guarantee that each discrete category had similar numbers of samples. Because of the relative subjectivity in determining these categories, three different sets of MIC thresholds were employed to assess the robustness of the results. All analyses were run four times to further assess robustness, and stability of which sites were identified. However, when doing so, the number of classification categories goes from two (influenza) to three (pseudomonas), without any statistical justification. To address this point, a more thorough search was performed using RRF - as RRF is much faster than $A B$. For this, the range of MIC values for each drug was discretized into bins of width 0.125 (on a $\log _{2}$ scale of MIC values), and two thresholds $\left(\theta_{1}, \theta_{2}\right)$ were defined, hereby dividing the distribution of MIC values into three domains. An initial classifier was then run for all combination of thresholds with $\theta_{1}<\theta_{2}$, and classification errors (RRF: Out-of-bag errors) were logged, and used to define MIC thresholds to perform the final analyses. A small chunk size (100) and a regular RF algorithm was used to speed up these computations (see Results).

\section{Additional file}

Additional file 1: Supplementary figures. (PDF 11,306 kb)

Abbreviations

AB: Adaptive boosting; ANCOVA: Analysis of covariance; CV: Cross-validation; GWAS: Genome-wide association study; MIC: Minimum inhibitory concentration; ML: Machine learning; RF: Random forest; RRF: Repeated random forest; SNP: Single nucleotide polymorphism

\section{Acknowledgements}

We thank Jeremy Dettman and Rees Kassen for sharing with us both their MIC and sequencing data, as well as the Center for Advanced Computing and Compute Ontario for providing us access to their servers. We are also grateful to Berthin Biyong, Graham Colby, Jeremy Dettman, Rees Kassen, and Matti Ruuskanen for discussions, and to two anonymous reviewers who helped improve this work.

\section{Authors' contributions}

SAB conceived the research; GSL, MH, JD and SAB wrote parts of the $R$ programming code; JD participated in method design and data handling; GSL and $\mathrm{MH}$ performed the data analyses. All authors wrote parts and edited the complete manuscript, before reading and approving the final manuscript. 


\section{Funding}

This work was supported by the University of Ottawa's Undergraduate Research Opportunity Program (GSL, MH) and assistantships (JD), and the Natural Sciences Research Council of Canada (SAB). Funding sources played no role in the design, results, or interpretations presented in this work.

\section{Availability of data and materials}

The data and source code used in this study are available from https://github. $\mathrm{com} /$ sarisbro (see LHDA_data.tar.bz2 in data folder).

\section{Ethics approval and consent to participate}

Not applicable. We have no human or animal data involved.

\section{Consent for publication}

Not applicable.

\section{Competing interests}

The authors declare that they have no competing interests.

\section{Received: 19 November 2018 Accepted: 21 May 2019} Published online: 10 June 2019

\section{References}

1. Stranger BE, Stahl EA, Raj T. Progress and promise of genome-wide association studies for human complex trait genetics. Genetics. 2011;187(2):367-83.

2. Lippert C, Sabatini R, Maher MC, Kang EY, Lee S, Arikan O, et al. Identification of individuals by trait prediction using whole-genome sequencing data. Proc Natl Acad Sci U S A. 2017;114(38):10166-71.

3. Visscher PM, Wray NR, Zhang Q, Sklar P, McCarthy MI, Brown MA, et al. 10 Years of GWAS discovery: biology, function, and translation. Am J Hum Genet. 2017; 101(1):5-22.

4. Visscher PM, Brown MA, McCarthy MI, Yang J. Five years of GWAS discovery. Am J Hum Genet. 2012;90(1):7-24.

5. Manolio TA, Collins FS, CoxNJ, Goldstein DB, Hindorff LA, Hunter DJ, et al. Finding the missing heritability of complex diseases. Nature. 2009;461(7265):747-53.

6. Cirulli ET, Goldstein DB. Uncovering the roles of rare variants in common disease through whole-genome sequencing. Nat Rev Genet. 2010;11(6): 415-25.

7. Risch N, Merikangas K. The future of genetic studies of complex human diseases. Science. 1996;273(5281):1516-7.

8. Kim BJ, Kim SH. Prediction of inherited genomic susceptibility to 20 common cancer types by a supervised machine-learning method. Proc Natl Acad Sci U S A. 2018;115(6):1322-7.

9. Hastie T, Tibshirani R, Friedman J. The elements of statistical learning. In: Overview of supervised learning. New York: Springer; 2009. p. 9-41.

10. Goldstein BA, Navar AM, Carter RE. Moving beyond regression techniques in cardiovascular risk prediction: applying machine learning to address analytic challenges. Eur Heart J. 2017;38(23):1805-14.

11. Angermueller C, Pärnamaa T, Parts L, Stegle O. Deep learning for computational biology. Mol Syst Biol. 2016;12(7):878.

12. Freund $Y$, Schapire RE, et al. Experiments with a new boosting algorithm. In: ICML. vol. 96; 1996. p. 148-56. https://scholar.google.com/scholar?q= Freund\%2C\%20Y.\%20\%26\%20Schapire\%2C\%20R.\%20\%281996\%29. \%20Experiments\%20with\%20a\%20new\%20boosting\%20algorithm\%2C \%20Machine\%20Learning\%3A\%20Proceedings\%20of\%20the \%20Thirteenth\%20International\%20Conference\%2C\%20148\%E2\%80 $\% 93156$.

13. Breiman $L$, et al. Arcing classifier (with discussion and a rejoinder by the author). Ann Stat. 1998;26(3):801-49.

14. Wolpert $\mathrm{DH}$. The lack of a priori distinctions between learning algorithms. Neural Comput. 1996;8(7):1341-90.

15. Zhou ZH. Ensemble methods: foundations and algorithms. Hoboken: CRC press; 2012

16. Shoji A, Aris-Brosou S, Culina A, Fayet A, Kirk H, Padget O, et al. Breeding phenology and winter activity predict subsequent breeding success in a trans-global migratory seabird. Biol Lett. 2015;11(10):20150671.

17. Liaw A, Wiener M. Classification and Regression by randomForest. R News. 2002;2(3):18-22. Available from: http://CRAN.R-project.org/doc/Rnews/.
18. Aris-Brosou S, Kim J, Li L, Liu H. Predicting the reasons of customer complaints: a first step toward anticipating quality issues of in vitro diagnostics assays with machine learning. JMIR Med Inform. 2018;6(2):e34.

19. Collaboration A, et al. The evolution of boosting algorithms-from machine learning to statistical modelling. Methods Inf Med. 2014;53(6):419-27.

20. Elith J, Leathwick JR, Hastie T. A working guide to boosted regression trees. J Anim Ecol. 2008;77(4):802-13.

21. Kamal RP, Katz JM, York IA. Molecular determinants of influenza virus pathogenesis in mice. In: Influenza Pathogenesis and Control-Volume I. Springer International Publishing; 2014. p. 243-74

22. Schrauwen EJ, de Graaf M, Herfst S, Rimmelzwaan GF, Osterhaus AD, Fouchier RA. Determinants of virulence of influenza A virus. Eur J Clin Microbiol Infect Dis. 2014;33(4):479-90.

23. Munita JM, Arias CA. Mechanisms of antibiotic resistance. In: Kudva IT, Cornick NA, Plummer PJ, Zhang Q, Nicholson TL, Bannantine JP, Bellaire BH, editors. Virulence Mechanisms of Bacterial Pathogens, Fifth Edition; 2016. p. 481-511.

24. Northrop Grumman Health ITVT J Craig Venter Institute. Influenza Research Database. 2017. Available from: https://www.fludb.org/brc/ home.spg?decorator=influenza. Accessed 24 May 2019.

25. Dettman JR, Rodrigue N, Aaron SD, Kassen R. Evolutionary genomics of epidemic and nonepidemic strains of Pseudomonas aeruginosa. Proc Natl Acad Sci U S A. 2013;110(52):21065-70.

26. Long JCD, Fodor E. The PB2 subunit of the Influenza A virus RNA polymeras e is imported into the mitochondrial matrix. J Virol. 2016;90(19):8729-38.

27. Llompart CM, Nieto A, Rodriguez-Frandsen A. Specific residues of PB2 and PA influenza virus polymerase subunits confer the ability for RNA polymerase II degradation and virus pathogenicity in mice. J Virol. 2014;88(6):3455-63.

28. Li J, Ishaq $M$, Prudence $M, X i X, H u T$, Liu Q, et al. Single mutation at the amino acid position 627 of PB2 that leads to increased virulence of an $\mathrm{H} 5 \mathrm{~N} 1$ avian influenza virus during adaptation in mice can be compensated by multiple mutations at other sites of PB2. Virus Res. 2009;144(1):123-9.

29. Yamaji R, Yamada S, Le MQ, Li C, Chen H, Qurnianingsih E, et al. Identification of PB2 mutations responsible for the efficient replication of H5N1 influenza viruses in human lung epithelial cells. J Virol. 2015;89(7): 3947-56.

30. Ellebedy $\mathrm{AH}$. Impact of adjuvants on the antibody responses to pre-pandemic H5N1 influenza vaccines: The University of Tennessee Health Science Center, Theses and Dissertations (ETD). Paper 75; 2011.

31. Bogs J, Kalthoff D, Veits J, Pavlova S, Schwemmle M, Mänz B, et al Reversion of PB2-627E to-627K during replication of an H5N1 Clade 2.2 virus in mammalian hosts depends on the origin of the nucleoprotein. J Virol. 2011;85(20):10691-8.

32. Sharshov K, Romanovskaya A, Uzhachenko R, Durymanov A, Zaykovskaya A, Kurskaya O, et al. Genetic and biological characterization of avian influenza H5N1 viruses isolated from wild birds and poultry in Western Siberia. Arch Virol. 2010;155(7):1145-50.

33. Hulse-Post D, Franks J, Boyd K, Salomon R, Hoffmann E, Yen H, et al. Molecular changes in the polymerase genes (PA and PB1) associated with high pathogenicity of $\mathrm{H} 5 \mathrm{~N} 1$ influenza virus in mallard ducks. J Virol. 2007;81(16):8515-24.

34. Taubenberger JK, Reid AH, Lourens RM, Wang R, Jin G, Fanning TG. Characterization of the 1918 influenza virus polymerase genes. Nature. 2005;437(7060):889-93.

35. Sugiyama K, Obayashi E, Kawaguchi A, Suzuki Y, Tame JRH, Nagata K, et al. Structural insight into the essential PB1-PB2 subunit contact of the influenza virus RNA polymerase. EMBO J. 2009;28(12):1803-11.

36. Gambaryan A, Robertson J, Matrosovich M. Effects of egg-adaptation on the receptor-binding properties of human influenza $A$ and $B$ viruses. Virology. 1999;258(2):232-9.

37. Godley L, Pfeifer J, Steinhauer D, Ely B, Shaw G, Kaufmann R, et al. Introduction of intersubunit disulfide bonds in the membrane-distal region of the influenza hemagglutinin abolishes membrane fusion activity. Cell. 1992;68(4):635-45.

38. Hensley SE, Das SR, Bailey AL, Schmidt LM, Hickman HD, Jayaraman A, et al. Hemagglutinin receptor binding avidity drives influenza A virus antigenic drift. Science. 2009;326(5953):734-6.

39. Takeda M, Leser GP, Russell CJ, Lamb RA. Influenza virus hemagglutinin concentrates in lipid raft microdomains for efficient viral fusion. Proc Natl Acad Sci. 2003;100(25):14610-7. 
40. Poole E, Elton D, Medcalf L, Digard P. Functional domains of the influenza A virus PB2 protein: identification of NP-and PB1-binding sites. Virology. 2004;321(1):120-33.

41. Li K, Guan Y, Wang J, Smith G, Xu K, Duan L, et al. Genesis of a highly pathogenic and potentially pandemic $\mathrm{H} 5 \mathrm{~N} 1$ influenza virus in eastern Asia. Nature. 2004;430(6996):209-13.

42. Gianfrani C, Oseroff C, Sidney J, Chesnut RW, Sette A. Human memory $C T L$ response specific for influenza $A$ virus is broad and multispecific. Human Immunol. 2000;61(5):438-52.

43. Min JY, Li S, Sen GC, Krug RM. A site on the influenza A virus NS1 protein mediates both inhibition of PKR activation and temporal regulation of viral RNA synthesis. Virology. 2007;363(1):236-43.

44. Hale BG, Randall RE, Ortín J, Jackson D. The multifunctional NS1 protein of influenza A viruses. J Gen Virol. 2008;89(10):2359-76.

45. Shin YK, Liu Q, Tikoo SK, Babiuk LA, Zhou Y. Influenza A virus NS1 protein activates the phosphatidylinositol 3-kinase (PI3K)/Akt pathway by direct interaction with the p85 subunit of PI3K. J Gen Virol. 2007;88(1):13-8.

46. Manzoor R, Sakoda Y, Nomura N, Tsuda Y, Ozaki H, Okamatsu M, et al. PB2 protein of a highly pathogenic avian influenza virus strain A/chicken/Yamaguchi/7/2004 (H5N1) determines its replication potential in pigs. J Virol. 2009;83(4):1572-8.

47. Leung BW, Chen H, Brownlee GG. Correlation between polymerase activity and pathogenicity in two duck H5N1 influenza viruses suggests that the polymerase contributes to pathogenicity. Virology. 2010;401(1):96-106.

48. Collin F, Karkare S, Maxwell A. Exploiting bacterial DNA gyrase as a drug target: current state and perspectives. Appl Microbiol Biotechnol. 2011;92(3):479-97.

49. Dalhoff A. Global fluoroquinolone resistance epidemiology and implictions for clinical use. Interdiscip Perspect Infect Dis. 2012;2012:976273.

50. Jones P, Binns D, Chang HY, Fraser M, Li W, McAnulla C, et al. InterProScan 5: genome-scale protein function classification. Bioinformatics. 2014;30(9):1236-40.

51. de Carvalho CCCR, Fernandes P. Siderophores as "Trojan Horses": tackling multidrug resistance? Front Microbiol. 2014;5:290.

52. Kos VN, McLaughlin RE, Gardner HA. The elucidation of mechanisms of ceftazidime resistance among clinical isolates of Pseudomonas aeruginosa using genomic data. Antimicrob Agents Chemother. 2016. Available from: http://aac.asm.org/content/early/2016/04/05/AAC.03113-15.abstract.

53. McGuffie BA, Vallet-Gely I, Dove SL. $\sigma$ factor and anti- $\sigma$ factor that control swarming motility and biofilm formation in Pseudomonas aeruginosa. J Bacteriol. 2015;198(5):755-65.

54. Schapire RE, Freund Y. Boosting: Foundations and algorithms. Cambridge: MIT press; 2012

55. Mayr A, Hofner B, Schmid M, et al. The importance of knowing when to stop. Methods Inf Med. 2012;51(2):178-86.

56. Yeung ATY Torfs, Jamshidi F, Bains M, Wiegand I, Hancock REW, et al. Swarming of Pseudomonas aeruginosa is controlled by a broad spectrum of transcriptional regulators, including MetR. J Bacteriol. 2009;191(18): 5592-602.

57. Amini S, Hottes AK, Smith LE, Tavazoie S. Fitness landscape of antibiotic tolerance in Pseudomonas aeruginosa biofilms. PLoS Pathog. 2011;7(10): e1002298.

58. Heeb S, Fletcher MP, Chhabra SR, Diggle SP, Williams P, Cámara M. Quinolones: from antibiotics to autoinducers. FEMS Microbiol Rev. 2011;35(2):247-74.

59. Nguyen AT, O'Neill MJ, Watts AM, Robson CL, Lamont IL, Wilks A, et al. Adaptation of iron homeostasis pathways by a Pseudomonas aeruginosa pyoverdine mutant in the cystic fibrosis lung. J Bacteriol. 2014;196(12): 2265-76.

60. Delcour AH. Outer membrane permeability and antibiotic resistance. Biochim Biophys Acta. 2009;1794(5):808-16.

61. Wozniak M, Tiuryn J, Wong L. An approach to identifying drug resistance associated mutations in bacterial strains. BMC Genomics. 2012;13(Suppl 7):S23.

62. Seyedmohammad S, Fuentealba NA, Marriott RAJ, Goetze TA, Edwardson JM, Barrera NP, et al. Structural model of FeoB, the iron transporter from Pseudomonas aeruginosa predicts a cysteine lined, GTP-gated pore. Biosci Rep. 2016;36(2):e00322.

63. Rouch DA, Cram DS, DiBerardino D, Littlejohn TG, Skurray RA. Efflux-mediated antiseptic resistance gene qacA from Staphylococcus aureus: common ancestry with tetracycline- and sugar-transport proteins. Mol Microbiol. 1990;4(12):2051-62.
64. Galle M, Carpentier I, Beyaert R. Structure and function of the Type III secretion system of Pseudomonas aeruginosa. Curr Protein Pept Sci. 2012;13(8):831-42.

65. Alvarez-Ortega C, Wiegand I, Olivares J, Hancock REW, Martínez JL. Genetic determinants involved in the susceptibility of Pseudomonas aeruginosa to beta-lactam antibiotics. Antimicrob Agents Chemother 2010;54(10):4159-67.

66. Kubacka A, Diez MS, Rojo D, Bargiela R, Ciordia S, Zapico l, et al. Understanding the antimicrobial mechanism of TiO?-based nanocomposite films in a pathogenic bacterium. Sci Rep. 2014;4:4134

67. Hall CW, Zhang L, Mah TF. PA3225 is a transcriptional repressor of antibiotic resistance mechanisms in Pseudomonas aeruginosa. Antimicrob Agents Chemother. 2017;61(8):e02114-16.

68. Srinivasan VB, Mondal A, Venkataramaiah M, Chauhan NK, Rajamohan G. Role of oxyRKP, a novel LysR-family transcriptional regulator, in antimicrobial resistance and virulence in Klebsiella pneumoniae. Microbiology. 2013;159(Pt 7):1301-14.

69. Laehnemann D, Peña-Miller R, Rosenstiel P, Beardmore R, Jansen G, Schulenburg H. Genomics of rapid adaptation to antibiotics: convergent evolution and scalable sequence amplification. Genome Biol Evol. 2014;6(6):1287-301.

70. Lee JM, Huddleston J, Doud MB, Hooper KA, Wu NC, Bedford T, et al. Deep mutational scanning of hemagglutinin helps predict evolutionary fates of human H3N2 influenza variants. Proc Natl Acad Sci U S A. 2018;115(35):E8276-85.

71. Ameur A, Wetterbom A, Feuk L, Gyllensten U. Global and unbiased detection of splice junctions from RNA-seq data. Genome Biol. 2010;11(3):R34.

72. Costa V, Aprile M, Esposito R, Ciccodicola A. RNA-Seq and human complex diseases: recent accomplishments and future perspectives. Eur J Hum Genet. 2013;21(2):134-42.

73. Torres-Oliva M, Almudi I, McGregor AP, Posnien N. A robust (re-)annotation approach to generate unbiased mapping references for RNA-seq-based analyses of differential expression across closely related species. BMC Genomics. 2016;17:392.

74. Leung MKK, Xiong HY, Lee LJ, Frey BJ. Deep learning of the tissue-regulated splicing code. Bioinformatics. 2014;30(12):i121-9.

75. Xiong HY, Alipanahi B, Lee $L J$, Bretschneider $H$, Merico D, Yuen RKC, et al. RNA splicing. The human splicing code reveals new insights into the genetic determinants of disease. Science. 2015;347(6218):1254806.

76. Basu S, Kumbier K, Brown JB, Yu B. Iterative random forests to discover predictive and stable high-order interactions. Proc Natl Acad Sci U S A. 2018;115(8):1943-8.

77. Stockwell DR, Peterson AT. Effects of sample size on accuracy of species distribution models. Ecol Model. 2002;148(1):1-13.

78. Stech O, Veits J, Weber S, Deckers D, Schröer D, Vahlenkamp TW, et al. Acquisition of a polybasic hemagglutinin cleavage site by a low-pathogenic avian influenza virus is not sufficient for immediate transformation into a highly pathogenic strain. J Virol. 2009;83(11):5864-8.

79. Bao Y, Bolotov P, Dernovoy D, Kiryutin B, Zaslavsky L, Tatusova T, et al. The influenza virus resource at the National Center for Biotechnology Information. J Virol. 2008;82(2):596-601.

80. van Riel D, Munster VJ, de Wit E, Rimmelzwaan GF, Fouchier RA, Osterhaus AD, et al. Human and avian influenza viruses target different cells in the lower respiratory tract of humans and other mammals. Am J Pathol. 2007;171(4):1215-23.

81. Subbarao K, Klimov A, Katz J, Regnery H, Lim W, Hall H, et al. Characterization of an avian influenza $A(H 5 N 1)$ virus isolated from a child with a fatal respiratory illness. Science. 1998;279(5349):393-6.

82. Edgar RC. MUSCLE: multiple sequence alignment with high accuracy and high throughput. Nucleic Acids Res. 2004;32(5):1792-7.

83. Winsor GL, Griffiths EJ, Lo R, Dhillon BK, Shay JA, Brinkman FSL. Enhanced annotations and features for comparing thousands of Pseudomonas genomes in the Pseudomonas genome database. Nucleic Acids Res. 2016;44(D1):D646-53.

84. Altschul SF, Gish W, Miller W, Myers EW, Lipman DJ. Basic loca alignment search tool. J Mol Biol. 1990;215(3):403-10.

85. Lawrence M, Huber W, Pagès H, Aboyoun P, Carlson M, Gentleman R, et al. Software for computing and annotating genomic ranges. PLoS Comput Biol. 2013;9(8):e1003118. 
86. Yotsuji A, Mitsuyama J, Hori R, Yasuda T, Saikawa I, Inoue M, et al. Mechanism of action of cephalosporins and resistance caused by decreased affinity for penicillin-binding proteins in Bacteroides fragilis. Antimicrob Agents Chemother. 1988;32(12):1848-53.

87. Kotra LP, Haddad J, Mobashery S. Aminoglycosides: perspectives on mechanisms of action and resistance and strategies to counter resistance. Antimicrob Agents Chemother. 2000;44(12):3249-56.

88. Alfaro E, Gámez M, García N. adabag: An R Package for Classification with Boosting and Bagging. J Stat Softw. 2013;54(2):1-35. Available from: http://www.jstatsoft.org/v54/i02/.

89. Liaw A, Wiener M. Classification and Regression by randomForest. R News. 2002;2(3):18-22. Available from: https://CRAN.R-project.org/doc/Rnews/.

90. R Core Team. R: A Language and Environment for Statistical Computing. Vienna, Austria; 2015. Available from: https://www.R-project.org/. Accessed 24 May 2019.

91. Microsoft, Weston S. foreach: Provides Foreach Looping Construct for R. 2017. R package version 1.4.4. Available from: https://CRAN.R-project.org/ package=foreach. Accessed 24 May 2019.

92. Melnyk AH, McCloskey N, Hinz AJ, Dettman J, Kassen R. Evolution of cost-free resistance under fluctuating drug selection in Pseudomonas aeruginosa. mSphere. 2017;2(4):e00158-17.

\section{Publisher's Note}

Springer Nature remains neutral with regard to jurisdictional claims in published maps and institutional affiliations.

Ready to submit your research? Choose BMC and benefit from:

- fast, convenient online submission

- thorough peer review by experienced researchers in your field

- rapid publication on acceptance

- support for research data, including large and complex data types

- gold Open Access which fosters wider collaboration and increased citations

- maximum visibility for your research: over $100 \mathrm{M}$ website views per year

At BMC, research is always in progress.

Learn more biomedcentral.com/submissions 